\title{
Collaborative Technologies in Solving Workplace Discrimination Against Women
}

\author{
Sangeeta Karmokar, Oluwaseun Adepoju \\ Auckland University of Technology, New Zealand \\ African Leadership University, Mauritius
}

\begin{abstract}
In a highly patriarchal society, women have been discriminated against in the workplace for long. Nigeria is a signatory to the convention on maternity protection which states that pregnant women can begin to take their maternity leaves 6 weeks before the delivery and 6 weeks after the baby is born. As much as labour policies are recorded and recognized on papers, reality stares us in the face every day as many organizations violate these laws that support and protect women in the workplace. There seems to be a huge difference between the labour laws that protect women in the workplace and what obtains in reality. It has also been observed that the reason why most organizations don't grant maternity leave opportunity to women is the vacuum they will leave during their maternity period. This qualitative expository paper seeks to unpack the realities of women discrimination in the Nigerian workplace on the grounds of maternity care and other challenges faced by the women. It also unpacked different ways of collaborative technologies that help solve these problems. A guided interview was conducted for randomly selected women but in the public and private sectors. The result showed a big difference in the implementation of maternity protection law in the public and the private sector. A consistent pattern of readiness for collaborative technologies in the workplace to help women navigate their maternity journey was observed. The study introduced a new framework named "Collaborative Cycle Technological Framework" to reimagine and facilitate ease of work for women.
\end{abstract}

\section{Introduction}

The expectations of women in the 21 st century are enormous. They are expected to thrive professionally and also perform maternity duties as mothers. No one can deny the fact that motherhood is a lot of work and women are meant to be given the opportunity to live a balanced life while they navigate the muddy waters of career and motherhood. The 9 months pregnancy period plays out differently for different women and there should be a level of understanding to help women navigate this period smoothly by employers, whether in the private or the public sector. It is high time employers stopped seeing pregnancy as a disturbance to women' effective performance at work and the idea of treating women as plagues during pregnancy should be highly discouraged. Women are not lazy and in-fact, psychology research has shown that women are better multi-taskers compared to the male folks. This study and other studies have established that women are more than willing to deliver optimally while on maternity leave if they have all the technology channels for virtual working put in place to remain productive.

In the 21 st century, technology is disrupting work life and physical presence in an office is becoming less and less important. Maternity duties and responsibilities should not put the professional lives of women on hold if collaborative technologies are involved. Depending on the type of job and role, different collaborative technologies can be used in helping women deliver on their roles. The use of emails for file exchange, zoom/skype/google hangout for video communication and telephony for information dissemination and delivery.

Today, there is exponential advancement in digital technologies. Over the past decade, technological advancement has caused disruption in many traditional industries. Car sharing, hire car services to disrupt the taxi industry, robots replace hundreds of blue-collared workers, mundane tasks are replaced by automation and even start-up companies with minimal experience compared to incumbent firms can triumph when armed with advanced technology [8]. The growth of transformative technology has changed human lives and the way they work. Advancement has brought immense convenience and benefits for both consumers, producers and businesses by bringing their productive capacity to a new height. This is considered as the fourth industrial revolution 
creating new innovations and markets across all fields such as biotechnology, health, food, travel, logistics, medical and entertainment.

The estimated economic impact of technologies across various applications will exceed $\$ 7$ trillion by 2025 [8]. This proves that disruptive technologies create growth or create entirely new markets through the introduction of products and services that are dramatically cheaper, better and convenient. It often disrupts workforce participation and offers revolutionary changes in the conduct of processes or operations [14]. Growth in technology has also provided tools and techniques for creating work-life balance and flexibility in working style.

Research and evidence indicate that flexible working hours lead to a balanced work-life for both employer and employees. It promotes healthier and more satisfied workforce, increased efficiency, reduced absenteeism, better health, reduced work conflict, higher job satisfaction and reduced work stress [23], especially for women. Although there are some organizations, with a good framework that supports flexible working hours for women, this is not practised widely.

\section{Guided Interview Questions}

Public Sector Interview

1. Are you aware of the Maternity Protection Labour Law in Nigeria?

2. What has been your experience as a woman working in the public sector in Nigeria?

3. Do you think the Public Sector workplace in Nigeria supports the rights of women?

4. Do you think you will function better if you are given the option of flexible work models with collaborative technologies during your maternity seasons?

Private Sector Interview

1. Are you aware of the Maternity Protection Labour Law in Nigeria?

2. What has been your experience as a woman working in the private sector in Nigeria?

3. Do you think the Private Sector workplace in Nigeria supports the rights of women?

4. Do you think you will function better if you are given the option of flexible work models with collaborative technologies during your maternity seasons?

\section{Literature Review}

This section will review important literatures on the maternity protection law in Nigeria, women as the future workforce and finally the advantages and limitations of flexible working structure for women.

\subsection{The Maternity Protection Law: ILO Act and Nigerian Reality}

According to the International Labour Organization (ILO), Maternity protection for women workers has been a core issue for the International Labour Organization since its foundation in 1919, when the governments, employers and trade unions of member States adopted the first Convention on maternity protection. Over the course of its history, the ILO's member States have adopted three Conventions on maternity protection (No. 3, 1919; No. 103, 1952; No. 183, 2000). These Conventions, together with their corresponding Recommendations (No. 95, 1952; No. 191, 2000) have progressively expanded the scope and entitlements of maternity protection at work and provided detailed guidance orienting national policy and action. The core concerns have been to enable women to successfully combine their reproductive and productive roles, and to prevent unequal treatment in employment due to their reproductive role.

The key reflections of the organization's most recent act on Maternity Protection, 2000 are to ensure that women's work does not pose risks to the health of the woman and her child and that women's reproductive roles do not compromise their economic and employment security. These elements include the right to:

- maternity leave;

- cash benefits to ensure the mother can support herself and her child during leave;

- medical care;

- protection of the health of pregnant and breastfeeding women and their children from workplace risks;

- protection from dismissal and discrimination; and

- breastfeeding on return to work.

The laws are on paper but a few sectors are adhering to the law. With the discovery in this study and other studies on Maternity Protection in Nigeria, the public sector is doing well in implementing the terms of the law but the same is not the case in the private sector. The ILO data on Maternity Leave and Benefit shows that Nigeria gives 12 weeks of maternity leave with $50 \%$ benefit and this based on employer's liability. When we compare this with Tanzania that gives 84days maternity leave, with a $100 \%$ benefit based on Social Security, Nigeria's Maternity Protection Law needs to be revisited.

\subsection{Women: The Future Workforce}


Over the past decade, despite rapid growth in the disruptive transformation of the globe, only a few studies have assessed the consequences of digitalisation on the workforce, especially for women. The digitalisation of the workspace has increased women's workforce participation via telecommuting and flexible work arrangements, outsourcing of projects and reduction in the time spent undertaking household labour [5]. Transformative technologies have increased the demand for cognitive and non-routine skills over manual and routine skills. There is some evidence that women are more efficient and experienced in non-routine analytical and interactive tasks when compared to men as a consequence of digitalisation and automation [22]. There are several factors contributing to the increase of women in workforces, such as increased number of women in higher education, cyclical labour market conditions, growth in service industries, government support for parenting leave, supportive childcare policies and increase in part-time/ flexible employment.

There have been dramatic changes in the landscape of digitised workforce. Women comprise nearly half of the workforce, with more families having dual earners. Part-time or flexibility in the workspace is one of the prominent factors for the increased number in women's participation. Growth in women's participation has increased demands for more flexibility in the workplace so that they can have more balanced work and life. The following section discusses the flexible working arrangement and its advantages and limitations in relation to women.

\subsection{Flexible Working Arrangement: Advantages and Limitations}

Flexible working arrangement (henceforth referred to as FWA) has two components - flexplace and flextime [1]. The former refers to 'where' the work gets done, while the latter refers to 'when' it is done. The flexibility in these two areas alleviate work-family conflict to a large extent and promotes a healthy work-life balance for a woman employee. Workplaces worldwide are moving towards compressed workweeks or encourage a results-only work environment [11]. The working days could be intense, but it ensures that the job at hand is completed and there are enough days left in the week to devote to non-work activities with family and friends.

There are only three finite resources that come into play when it comes to work or family. These are time, attention and energy [1], that a working woman has to stretch in every possible direction to meet the demands at work and the expectations at home. In most of the families, man is considered as a bread-winner of his family, a modern woman who wants to be financially independent has to perform a dual role of breadwinner and managing domestic chores. As a result, these resources, especially for women, are in high demand, as both work and family vie for them. FWA helps allocate these resources evenly, leading to the employee's personal development and fostering a positive job attitude. FWA is also known to lower turnover intentions [18], which works just fine as women prefer stability in every aspect of their lives. In fact, FWA makes employees proactive, who come up with innovative solutions to problems - perhaps the same way they tend to domestic responsibilities. They usually work by themselves at home and so have to figure out solutions to problems on their own. This improves their cognitive skills, making them self-sufficient and efficient.

When working from the comfort of home, it is quite easy to get distracted by never-ending household chores or entertainment options, as a relief from the chores. Under these circumstances, practising self-control and following a rigid schedule becomes essential. This is not everyone's cup of tea. Often, this results in blurring the roles of family and work, giving rise to trust issues on the part of the employer. The feeling of isolation experienced when working from a remote location can easily become demotivating, spurring the urge to engage in activities that are non-work related, which can actually work as a stimulant. If the line manager is not accustomed to such outcomes of FWA, this can fuel mistrust, leading to the breakdown of employer-employee relationships. More often than not, these managers are men who are not comfortable around working women with families.

The conservatives also argue that FWA increases individualism and limits collaboration. But if we consider work to be the 'economic' and family to be the 'social' pillars of our lives [3], we would invariably find that the two are intertwined for working women with family responsibilities - one does not exist without the other. This understanding should reduce the tension between the two, by giving each its due time. Research has shown that female employees who are offered FWA have higher levels of job satisfaction and commitment towards their employers when compared to their non-flexible counterparts [21]. These employees respond to FWA "by exerting additional effort, in order to return benefit to their employer" [13]. It is interesting to note here that giving back earnestly is a quality associated largely with women. FWA also helps to be productive in the hours spent travelling through congested traffic, gives an alternative to overloaded facilities at the workplace, as well as brings panacea to counterboring and repetitive work 
[7]. In this way, FWA raises morale and decreases absenteeism among the female workforce.

\section{Methodology}

This study is expository qualitative research. A number of randomly selected women, that represents both the private and the public sector were interviewed in a structured interview session. Interview reports were analysed with opinion analytics and recurring idea spectrum.

\section{Discussion}

This section discusses the qualitative interview report of women in the public sector and the qualitative interview reports of women working in the private sector. The insights from the interviews are corroborated with the reviewed literature.

\subsection{Qualitative Interview Reports: Women working in the Public Sector}

These interview reports show the responses from randomly selected women working with the government across the three organograms of the government (Federal, state and local government). They also represent different roles and professional cadres. The following were the questions asked during the guided interview:

- Are you aware of the Maternity Protection Labour Law in Nigeria?

- What has been your experience as a woman working in the public sector in Nigeria?

- Do you think the Public Sector workplace in Nigeria supports the rights of women?

- Do you think you will function better if you are given the option of flexible work models with collaborative technologies during your maternity seasons?

Summarizing the responses of PUS/001/FG, a 50-year-old woman who works for one of the Federal Ministry Offices had this to say:

"I have worked for the federal government for 25 years and I have never had any issues whenever I had a baby. I have never seen the Maternity Protection Law but I do not have any issues in my own place of work. I have three children and I had written letters to my immediate superior to take maternity leave from the 6th month of pregnancies and they were all granted. My leaves have been paid and they often call me from the office when they need a piece of information from me. I either quickly show up in the office or I give them the information on the phone or through emails. I resume back to work after three months of nursing".

The responses from this woman show that even though she might not have seen or read the Maternity Protection Law in Nigeria, she had no problem with maternity leaves and she was getting her pay during the leave period. Her responses also showed that she had the flexibility of taking even more maternity leave compared to the one stated in the Maternity Protection Law. She also noted that she used telephony and Email technology to deliver tasks while she was away from work. This is an indication that with a more deliberate effort in technology investment, women can take ample maternity leave time and still deliver on their roles as if they never left the office physically.

In another interview with PUS/002/SG, a secondary school teacher with the Oyo state government, below is her response:

"I have never seen the Maternity Protection Law but as a teacher, but I know my school supports pregnant teachers and they go on leave when they are approaching their due dates. I am not married and have never gone on maternity leave but I had taken sick leaves and professional travel leaves. The problem with teachers going on leave is the vacuum teachers leave because of the absence of other subject matter experts that can take up their subjects but we have always found a way around it. The length of maternity leave for women is also dependent on the generosity of the principal of the school. Some principals release pregnant teachers as soon as they have become too heavy to function well in the classroom. However, I really wish Online Learning Management Systems can be introduced so that women on maternity leave can still deliver lectures online while waiting for delivery".

This response also showed flexibility in the Maternity leave for women working in the public sector. It seems it does not matter whether the Maternity Protection Law existed or not, as long as women can take maternity or sick leaves when they need to, the situation is fine. This woman also mentioned that if Learning Managements Systems are introduced, it can increase the flexibility of work for women whenever they are away.

PUS/003/LG, a 40-year-old Local government secretariat secretary had this to say:

"I don't think women are discriminated against in our local government office. Women have equal rights in the office and we can take leave when we need to. It is not a problem to get pregnant here and I gave birth to two children while on this job. I was given enough time to take care of my babies before I resumed back at work. We have the culture of someone standing in for each other in case someone 
is not available. My role requires that I come to the office physically so I am not sure if disruptive technologies will help me deliver on my role more effectively or not".

This response again points in the direction of flexibility on Maternity leaves and freedom of decisions in the workplace. This woman was not sure whether technology will make her more effective during her time away from work or not but she appreciated the culture of "people standing in for people" at her place of work.

\subsection{Qualitative Interview Reports: Women working in the Private Sector}

This interview report shows the responses from randomly selected women working or have been discriminated against in the Private Sector. They also represent different roles, companies and professional cadre. The following were the questions asked during the guided interview:

- Are you aware of the Maternity Protection Labour Law in Nigeria?

- What has been your experience as a woman working in the private sector in Nigeria?

- Do you think the Private Sector workplace in Nigeria supports the rights of women?

- Do you think you will function better if you are given the option of flexible work models with collaborative technologies during your maternity seasons?

PV/001/ENT, a 31-year-old entrepreneur in Lagos state Nigeria had this to say:

"I applied to a popular bank in Nigeria as a contract marketing staff and I was invited for a physical interview. I found it ridiculous that I was asked if I was pregnant. I queried the interviewer that asked me the question what difference pregnancy would make on their decision to employ me, I was shocked when he said and I quote "we don't want someone with a big belly roaming the streets to look for clients'. I found the comment insulting and demeaning. I left the interview room angry and offended. I obviously did not get the job because I challenged the oppressive policy of the bank".

This response shows that the private sector's experience is different from what we have recorded so far in the public sector. Diana Ibori, an author at Legit.ng, in an article he published in 2018 titled "How maternity leave work in Nigeria" reported that Maternity Protection Law is a global labour treaty that Nigeria signed like any other country but has not been domesticated in the Nigerian National
Assembly. This may be the reason why it is difficult to challenge such discrimination in the court of law.

The expectations of the bank are for the sales assistants to get people to open bank accounts and it should not matter whether they get them online or offline. This is where the role of disruptive technologies comes into play.

PV/002/CRP, a 36-year-old woman working in the corporate Lagos, Nigeria had this to say:

'In my last job, I was made to sign an agreement that I would not get pregnant on the job and I would need to resign or take a leave without pay if I ever want to get pregnant. I found the agreement inappropriate but I needed to secure key economic stability at the point in time so I decided to work there for two full years and then I resigned when I decided to have my first child. Many private sector businesses in Nigeria are like this and I believe something must be done about it soonest. The most surprising situation for me was that the wife of the Managing Director of the company that made the agreement not to get pregnant was pregnant at the time I started with the company and she works for a multinational company in Lagos with pregnancy.

In my current place of work, there was no strict agreement on not getting pregnant but it was stated on the contract that maternity leave is without pay'.

This report shows a new dimension to this issue. It is still discrimination to state on contracts that women can go on maternity leave but without pay. In another interview with PV/003/STUP, a 40-yearold woman who is a co-founder of a big startup company in Port-Harcourt Nigeria, she narrated the situation below:

"I resigned my job in consulting in 2015 when my boss was always picking on me as lazy because I was pregnant. The emotional trauma I was going through was too much so I resigned. I stayed off a career for four years to have all my children before I started looking for new jobs. Staying off my career passion for four years negatively affected me when I returned as it was very hard for me to get in my early career positions because of my age. I was lucky to eventually get to work for a startup company that gave me the flexibility to take care of my family and work at the same time. I am now a shareholder in the same company and I would not trade this for anything. This opportunity that I enjoyed shaped our organization culture and our staff can decide to work remotely as long as they deliver on their tasks daily. We use Slack for internal communications and Zoom for team check-in".

This report shows again the blessedness of flexible working culture and the relevance of 
collaborative technologies for women undergoing their maternity journeys.

PV/004/NGO, a policy researcher had this to say:

"As a policy researcher for a Non-governmental organization, I have been leading a team of five and we have never met physically. We have done most of our health policy briefs and reports through collaborative writing on Google Doc and Canva. I believe technology is an enabler and it must be maximized for productivity. I have flexible work hours and as a matter of fact, I still had a team check-in with my team on google hangout the day I gave birth to my last child. That is how powerful technology is".

\section{Recommendations}

\subsection{Collaborative Cycle Technological Framework}

In view of supporting and remodelling the workplace for women and accommodating shifting family patterns and to reduce workplace conflict, we have developed a three-pronged Collaborative Cycle Technological framework. It is built by innovating and adaptation of existing frameworks that are already in practice in contemporary workplaces.

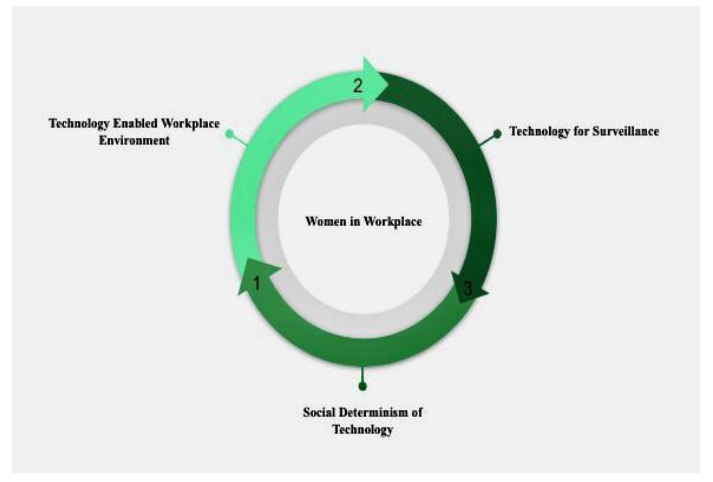

Figure 1. Collaborative Cycle Technological Framework

\subsection{Technology for surveillance}

The very first cog of the framework involves a shift in work culture among senior management. Emphasis should be on building trust among employees by hiring efficient and responsible staff who are held accountable for their share of the workload. Collaborative technology, a keen blockchain technology, can further this cause because the new capabilities it enables prompt societal change. Although considered a technology that is set to transform the world of financial transactions, the same concept of "peer-to-peer. distributed ledger" of blockchain can be applied to non-financial exchanges as well, where everyone can see the others entry in real-time [19]. This prevents any one person from tricking the system and thereby fosters mutual trust.

An open-source software, blockchain operates by sharing information among collaborators and prevents users from erasing any information that's on the system [4]. A decentralised mechanism in the workplace, it could operate on "distributed consensus" (Ibid.) informing colleagues about an act that has been completed. This update, once recorded, would be irreversible. The knowledge of such a system would make the employee on FWA aware of her responsibilities and make her accountable to others in the team.

Any social, ethical, moral issues that might arise from such a flexible working arrangement would lead us back to developing trust within the organisation and among the employees. Technology-enabled surveillance can enable this to a large extent. In fact, this form of technology can help overcome space constraints at the workplace by encouraging staff to work from home and thereby keep overheads to a minimum with the existing facilities going a long way.

\subsection{Social determinism of technology}

Use of appropriate technology is largely determined by the social arrangement around it. In other words, the social determinism of technology [15] helps identify the most appropriate device or internet application for FWA. This brings us to the second cog of the framework, whereby it is the users who decide which technology is most relevant to get the job completed. About a decade ago, G Suite was the chosen platform for corporate organisations. It was used not only for linear communication via emails or chats for an immediate response but also for collaboration using Google docs and the shared folders. Meetings were conducted on Hangouts, using VOIP and video calls. These paved the way for big data and cloud storage services, which meant employees could access work files from just about anywhere.

Lately, the preferred platform with most organisations appears to be Microsoft's revamped Office 365. One Drive is used for cloud storage as well as for document sharing, while SharePoint works as a repository of folders and files. Skype, also owned by Microsoft, makes up for the VOIP (video and voice over IP) platform, useful for meetings and video calls. Rumour has it that Skype will be replaced by Office Teams in the not very distant future. There are other regular features, like the Calendar which acts as a diary of meetings and appointments, and the PowerPoint and Word. 
The choice between Google and Microsoft, of course, depends on the nature of work the organisation engages in. If the work is predominantly collaborative, then G Suite is the best option. But if the focus of the organisation is primarily on the use of "powerful and sophisticated features" then Office 365 would out-weigh Google by a big margin. Thus, it is imperative for the employers, along with employees, to determine which technology would work to their advantage when it comes to implementation of FWA.

\subsection{Technology-enabled FWA}

When compared to a regular employee on a regular work shift, a woman on FWA is often perceived as an unambitious employee, not very keen on career advancement (Rogier et al, 2004). If the managers go beyond such stereotypes and examine the capabilities of both of these employees, they might be surprised to learn about the dedication of the employee on FWA, for reasons mentioned above. Thus, the third cog of the framework is with regard to improvement in working arrangements. With the rapid changes in work environments and competitive salaries, "job enrichment has become a fundamental tool for management in improving employee's motivation and organizational growth" [2]. Money is no longer the ultimate decisive factor, rather a host of other things that pertain to the work culture in the organisation that plays an increasingly prominent role. [2] has listed them as follows:

- Giving more freedom.

- Encouraging participation.

- Giving employees the freedom to select the method of working.

- Allowing employees to select the place at which they would like to work.

- Allowing workers to select the tools that they require on the job.

- Allowing workers to decide the layout of a plant or office.

In keeping with these guidelines, we recommend the following pointers to improve the work environment for women on maternity care.

- Flextime: Vary the beginning and end of the workday.

- Extended leave: Sabbaticals at regular intervals. They could be paid or unpaid leave for personal reasons.

- Part-time work: The option to work less than 40 hours a week.

- Compressed workweek: Fulfilling work week obligations in fewer than five days.

- Telecommuting: Some work taking place outside of the office.
- Work from home: All working hours carried out from home.

- Job Share: Two people sharing the job of one, by dividing the hours and responsibilities between them.

\section{Conclusions}

In recent decades, women workforce participation has increased. The knowledge economy has given opportunities for career flexibility, which can clearly be beneficial for women who have children and other responsibilities. With the rapid growth in technology and change in the lifestyle flexible working is becoming popular. Technological transformation has reformed the time, space and style of our work. A lot of organizations are offering flexible working arrangements because of its benefits to both employer and the employee. The proposed Collaborative Cycle Technological framework may result in increased job satisfaction and productivity as women various constraints imposed by household contribution.

\section{References}

[1] Allen, T. D., Johnson, R. C., Kiburz, K. M. \& Shockley, K. (2013). Work-family Conflict and Flexible Work Arrangements: Deconstructing Flexibility. Personnel Psychology, 66, 345-376.

[2] Choudhary, S. (2016). Job Enrichment: A Tool for Employee Motivation. International Journal of Applied Research, 2(5), 1020-1024.

[3] Crompton, R. (2002). Employment, Flexible Working and the Family. British Journal of Sociology, 53(4), 537558 .

[4] Crosby, M., Nachiappan, Pattanayak, P., Verma, S. \& Kalyanaraman, V. (2016). BlockChain Technology: Beyond Bitcoin. Applied Innovation Review.

[5] Dettling, L. J. (2017). Broadband in the Labor Market: The Impact of Residential High- speed Internet on Married Women's Labor Force Participation. ILR Review, 70(2), 451-482.

[6] Development, O. f. E. C. a. (2018). Transformative Technologies and jobs of the Future [Press release].

[7] Elbing, A. O., Gadon, H. \& Gordon, J. R. M. (1975). Flexible working Hours: The Missing Link. Harvard Business Review, 52 (1), 18-33.

[8] En, C. F. (2017). The Impact of Disruptive Technologies. Hwa Chong Institute.

[9] Greene, L., \& Mamic, I. (2015). The Future of Work: Increasing Reach Through Mobile Technology [Press release]. 
[10] Harell, G., \& Daim, T. U. (2009). Virtual Teams and the Importance of Building Trust.

[11] Hornung, S., Rousseau, D.M. \& Glaser, J. (2008). Creating Flexible Work Arrangements Through Idiosyncratic Deals. Journal of Applied Psychology, 93(3), 655-664.

[12] Karmokar, S. (2019). Transformative Technologies for Social Change. Paper presented at the 14th European Conference on Innovation and Entrepreneurship, Kalamata, Greece.

[13] Kelliher, C., \& Anderson, D. (2010). Doing more with less? Flexible working practices and the intensification of work. Human Relations, 63(1), 83-106.

[14] Kostoff, R. N., Boylan, R., \& Simons, G. R. (2004). Disruptive Technology Roadmap. Technological Forecasting \& Social Change (71), 141-159.

[15] Lievrouw, L. A. \& Livingstone, S. M. (2006). Handbook of New Media: Student Edition. Sage Publications: London.

[16] L.Jarvenpaa, S., \& Ives, B. (1996). Introducing Transformational Information Technologies: The Case of the World Wide Web Technology. International Journal of Electronic Commerce, 1, 95-126.

[17] Manyika, J., Lund, S., Auguste, B., Mendonca, L., Welsh, T., \& Ramaswam, S. (2011). An Economy that Works: Job Creation and America's Future.

[18] McNall, L. A., Masuda, A. D. \& Nicklin, J. M. (2009). Flexible Work Arrangements, Job Satisfaction, and Turnover Intentions: The Mediating Role of Work-toFamily Enrichment. Journal of Psychology: Interdisciplinary and Applied, 144(1), 61-81.

[19] Mearian, L. (2019). What is blockchain? The complete guide. Computer World, New Zealand, January 30.

[20] Rogier, S. A. \& Padgett, M. Y. (2004). The impact of utilizing a flexible work schedule on the perceived career advancement potential of women. Human Resource Development Quarterly, 15(1), 89-106.

[21] Terri, A. S. \& Lankau, M. J. (1997). Relationships of gender, family responsibility and flexible work hours to organizational commitment and job satisfaction. Journal of Organisational Behaviour, 18(4), 377-391.

[22] Watson, T., Corliss, M., \& Le, M. (2018). Digitalisation and Women's Workforce Participation in the Indo-Pacific. Australian Journal of Labour Economics, 21(1), 45-74.

[23] Wheatley, D. (2017). Employee Satisfaction and Use of Flexible Working Arrangements. Work, Employment and Society, 3(4), 567-585. 